\title{
Numerical Aspects and Performances of Trajectory Planning Methods of Flexible Axes
}

\author{
Jean-Yves Dieulot, Issam Thimoumi, Frédéric Colas, Richard Béarée
}

\begin{abstract}
Adequate Path Planning design is an important stage for controlling flexible axes because it may allow to cancel vibrations induced by oscillating modes. Among bang-bang profiles which are linked to optimal control, jerk assignment (acceleration derivative) and input shapers have been investigated. Theoretical results show the performance and robustness with respect to natural frequency mismatch. Practical validations on a real robot arm show the relevance of the jerk algorithm which is more robust with the same productivity performances as input shaping techniques.
\end{abstract}

Keywords: Flexible axes, Vibrations, bang-bang laws, Input shapers, Motion Planning.

\section{Introduction}

Vibration control of flexible Cartesian robots or axes is strongly related to productivity. Indeed, lighter structures allow material savings and higher performances in terms of speed, acceleration... which, in turn, cause undesirable oscillations. In practice, classical servo control fails to reduce vibrations, which, in many cases, restrains the operating speed [1]. The only way to damp the oscillations consists of designing proper smooth reference trajectories, which should be however as fast as possible [2]. Many methods have been introduced using the inversion motion equations or a combination of smooth trigonometric or polynomial functions, e.g. [3]. Very often, though, these methods face the robustness problem with respect to uncertainties in the dominant frequency, which is the main model parameter to be taken into account into anti-vibration algorithms design. Near-optimal bang-bang methods have been designed to solve the trade-off between productivity and vibrational behavior. These mainly consist of convolving a series of impulses with the control input, where the inter-pulse duration is adequately chosen in a way that the resulting oscillations are in phase opposition [4]. These techniques are known as input shapers, and have proven to be quite effective on linear systems e.g. [5, 6]. On another hand, the most popular damping scheme consists of a bang-bang profile in jerk (which is the derivative of acceleration), and has been proven to be related to input shapers with negative weights [7]. The goal of the paper is to compare different bang-bang laws (shapers, jerk...) in terms of productivity and sensitivity to parameter uncertainty, for a classical model of flexible axis controlled in closed-loop. In a first part, different trajectory planning methods are presented and theoretical results, in terms of vibration attenuation and robustness, are presented. The second part deals with an experimental validation on a real industrial pick-and-place robot which will draw a fair comparison in terms of productivity between these methods.

\section{Preshaping laws}

\subsection{Architecture of $\mathrm{CNC}$ axes}

Control of a CNC axis or a Cartesian robot arm is decomposed into three phases [1]:

- geometric motion planning which provides the desired path within the whole workspace (in 2- or 3-D) 


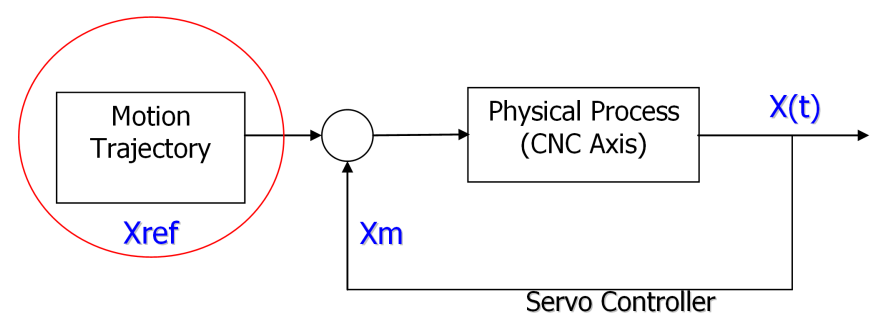

Figure 1: Control strategy of a CNC Axis

- a time-dependent path planning stage which should, of course, take into account for saturations, but, which will be shown later, should be designed to cancel undesirable vibrations

- a servo controller which should help to reject disturbances and to track the reference trajectory.

In practice, the model of a flexible axis controlled with cascaded loop will be [1]

$$
\frac{x(s)}{x_{r e f}(s)}=\frac{1+\frac{k f_{v}}{k_{v}} s}{1+\frac{s}{k_{v}}} \frac{1+\frac{2 \zeta}{\omega} s}{1+\frac{2 \zeta}{\omega} s+\frac{1}{\omega^{2}} s^{2}}
$$

where $x$ is the position of the motor, $x_{r e f}$ is the reference trajectory, $k_{v}, k f_{v}$ are servo gains (position and speed gains, respectively), $\zeta, \omega$ are respectively the damping ratio (assumed as very small, $\zeta<<1$ ) and the natural frequency of the axis modeled as a two-mass-spring damper unit. The main purpose of this paper will be the design of the time-dependent path-planning of the closed-loop system represented by equation (1).

\subsection{Smooth continuous laws}

A first way of designing the reference trajectory, would be to invert the whole path planning, such as in the so-called flatness approach [3]. In practice, though, model parameters vary since the structure consists of an assembly of flexible beams. The linear approximation of equation (1) is indeed valid for one set-point, which is the case during the short periods corresponding to the start and stop procedures, but not during the whole course. For point-to-point displacements, a stage of acceleration and deceleration is necessary. In CNC control, these stages are driven in a very sharp way, commonly a bang-bang in acceleration, which consequently excites the flexible mode of equation (1) and generates high-amplitude vibrations. Smooth trajectories based upon the natural frequency of the closed-loop system (1) can thus be designed, and an obvious method consists of introducing such laws as trigonometric trajectories (Fig. 2).

The maximal vibratory error for a "sine" profile is

$$
\left(\varepsilon_{v i b}\right)_{\max }=\bar{x}_{r e f} \frac{\sin \left(\frac{\pi \tau}{2}\right)}{\pi \tau\left(\tau^{2}-1\right)}
$$

for a square sine [2] profile ( $\tau=T_{f} \omega / 2 \pi ; T_{f}$ is the motion duration, $\bar{x}_{r e f}$ is the target position). In practice, the error is significantly reduced for a movement of duration superior to 4 times the natural period. An alternative consist of building smooth polynomials which would verify smooth constraints on the dynamics such as minimizing the amplitude of the jerk during the whole displacement, the corresponding polynomial is:

$$
x_{r e f}=\frac{30 t^{3} \bar{x}_{r e f}}{T_{f}^{5}}\left(\frac{t^{2}}{5}-\frac{T_{f} t}{2}+\frac{T_{f}^{2}}{3}\right)
$$




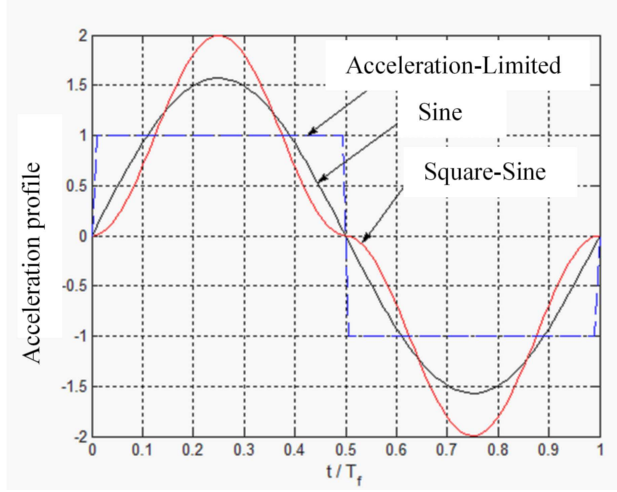

Figure 2: Smooth Control Laws

and the corresponding maximum error decreases again after a few periods [2]:

$$
\left(\varepsilon_{v i b}\right)_{\max }=\frac{15 \bar{x}_{r e f}}{\pi^{5} \tau^{5}}\left[3 \pi \tau \cos (\pi \tau)+\left(-3+\pi^{2} \tau^{2}\right) \sin (\pi \tau)\right]
$$

\subsection{Jerk Laws}

Contrary to previous laws, it is also possible to use "bang-bang" or discontinuous laws to achieve the feedforward control of CNC axes, which are related to time-optimal control as proven in [8].

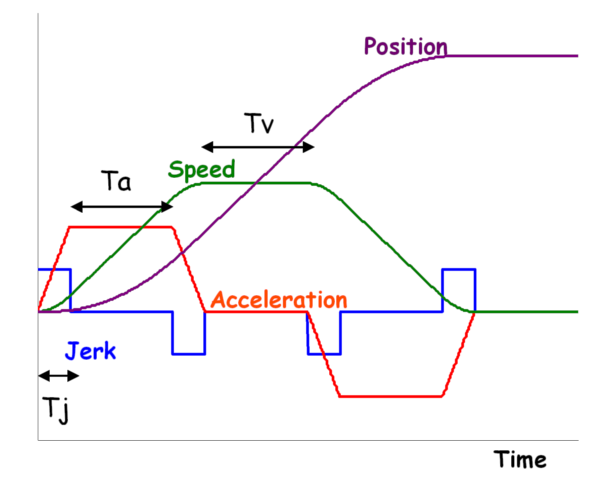

Figure 3: Bang-Bang Profile (Jerk-limited)

The maximum theoretical error for a bang-bang in acceleration is $\frac{\max _{t \geq T f}(\varepsilon(t))}{\bar{x}_{r e f}}=\left(\operatorname{sinc}\left(\frac{\pi \cdot \tau}{2}\right)\right)^{2}, \tau=$ $T_{f} \omega / 2 \pi$. For a profile with a bang-bang in jerk, one obtains [2]:

$$
\max _{t \geq T_{f}}\left|\frac{\varepsilon(t)}{\bar{x}_{r e f}}\right|=\frac{4 A_{m}}{\omega^{2}} \cdot \sqrt{\frac{1+k f_{v}^{2} \omega^{2} / k_{v}^{2}}{1+\omega^{2} / k_{v}^{2}}} \cdot\left|\operatorname{sinc}\left(\frac{T_{j} \omega}{2}\right)\right| \Psi\left(T_{j}, T_{a}, T_{v}\right)
$$

where $T_{f}=4 T_{j}+2 T_{a}+T_{v}$ (see Fig. 3 ), $A_{m}$ is the maximum acceleration, and:

$$
\Psi\left(T_{j}, T_{a}, T_{v}\right)=\left|\sin \left(\left(T_{a}+T_{j}\right) \omega / 2\right) \sin \left(\left(T_{a}+2 T_{j}+T_{v}\right) \omega / 2\right)\right|
$$

In theory, it is possible to find a value of Jerk for zero-vibration which will be:

$$
T_{j}=\frac{2 \pi}{\omega} \text { and } \mathrm{J}=\frac{2 \pi}{\omega} \mathrm{A}_{\mathrm{m}}
$$

The theoretical robustness with respect to an uncertainty in the natural period $T$ is shown below: 


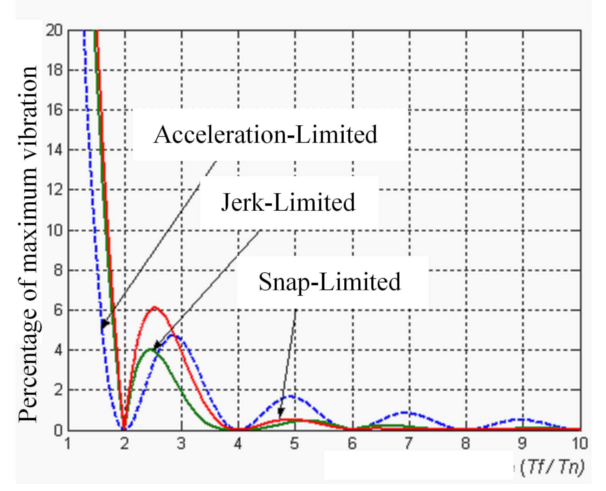

Figure 4: Residual vibrations

\subsection{Input Shapers}

The principle of input shapers was introduced by Singer and Seering [4] and consists of convolving the reference or the input with a series of well-chosen impulses. Jerk motion planning can be considered as input shaping with negative weights. The underlying idea is that the oscillation will be compensated

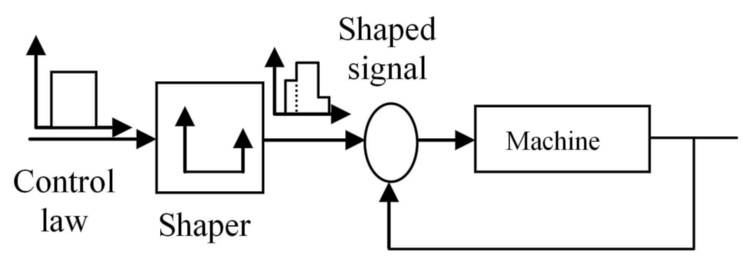

Figure 5: Input Shaper Principle

by that induced by a shifted pulse as shown Fig. 6: A shaper is thus a series of shifted pulses which can

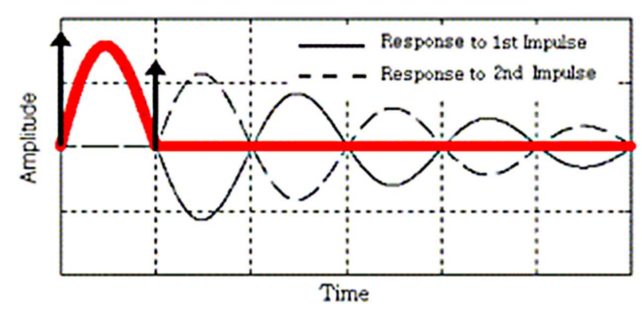

Figure 6: Superposition of oscillations

be written

$$
F(s)=A_{0}+\sum_{i=1}^{n} A_{i} e^{-s T_{i}}
$$

where $\sum_{i=1}^{n} A_{i}=1$. The response to a second order system will be:

$$
x(t)=\sum_{i=1}^{n} \bar{x}_{r e f}\left[\frac{A_{i} \omega}{\sqrt{1-\zeta^{2}}} e^{-\zeta \omega\left(t-T_{i}\right)}\right] \sin \left(\omega \sqrt{1-\zeta^{2}}\left(t-T_{i}\right)\right)
$$


- the Zero-Vibration (ZV) shaper, which is a two pulse filter for which the vibration at the stop stage should be zero; one obtains the following equations:

$$
\left\{\begin{array}{l}
A_{0}+A_{1} e^{\zeta \omega T_{1}} \cos \left(\omega \sqrt{1-\zeta^{2}} T_{1}\right)=0 \\
A_{1} e^{\zeta \omega T_{1}} \sin \left(\omega \sqrt{1-\zeta^{2}} T_{1}\right)=0 \\
A_{0}+A_{1}=1
\end{array}\right.
$$

- the Zero-Vibration Derivative (ZVD) shaper which is a three pulse filter for which the position and its derivative at the stop stage should be zero; equations are:

$$
\left[\begin{array}{c}
A_{i} \\
T_{i}
\end{array}\right]=\left[\begin{array}{ccc}
\frac{1}{D(\zeta)} & \frac{2 e^{\frac{-\zeta \pi}{\sqrt{1-\zeta^{2}}}}}{D(\zeta)} & \frac{e^{\frac{-2 \zeta \pi}{\sqrt{1-\zeta^{2}}}}}{D(\zeta)} \\
0 & \frac{\pi}{\omega \sqrt{1-\zeta^{2}}} & \frac{2 \pi}{\omega \sqrt{1-\zeta^{2}}}
\end{array}\right]
$$

where $D(\zeta)=1+2 e^{\frac{-\zeta \pi}{\sqrt{1-\zeta^{2}}}}+e^{\frac{-2 \zeta \pi}{\sqrt{1-\zeta^{2}}}}$

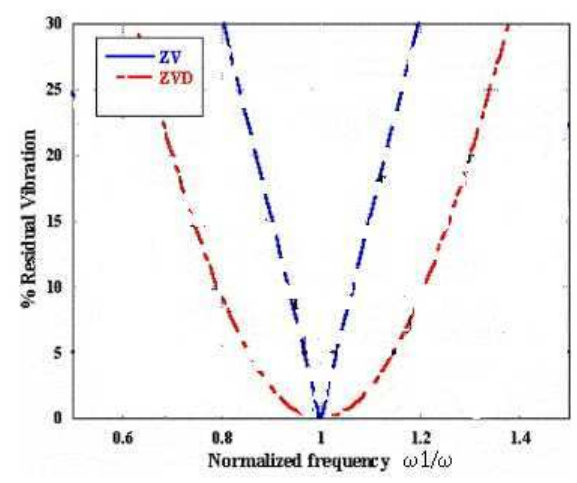

Figure 7: Shaper robustness

Figure 7 shows shaper robustness where the percentage of residual vibration is plotted versus the ratio $\omega_{1} / \omega$ where $\omega_{1}=2 \pi / T_{1}$ (a Specified Insensitive is a 4-pulse shaper [9]). The more pulses, the more the shaper will be robust with respect to natural frequency uncertainty. However, it can be understood that adding pulses leads to higher cycle times, as will be shown later on. One can see that the ZV shaper, which is quite performing, is not really robust with respect to natural frequency mismatch.

When applied to equation (1), the ZV shaper gives a maximal error of:

$$
\max _{t \geq T_{f}}\left|\frac{\varepsilon(t)}{\bar{x}_{r e f}}\right|=\frac{A}{2+2 T^{2} \omega^{2}} \sqrt{\left(\begin{array}{l}
\left(1+\cos \left(\frac{\pi \omega}{\omega_{1}}\right)-T \omega \sin \left(\frac{\pi \omega}{\omega_{1}}\right)\right)^{2} \\
+\left(T \omega+T \omega \cos \left(\frac{\pi \omega}{\omega_{1}}\right)+\sin \left(\frac{\pi \omega}{\omega_{1}}\right)\right)^{2}
\end{array}\right)}
$$

with a theoretical cycle time of:

$$
T_{f}=\frac{1}{2}\left(\sqrt{\frac{16 \bar{x}_{r e f}}{A}}-T_{1}\right)
$$

The ZVD shaper gives a maximal error of

$$
\max _{t \geq T_{f}}\left|\frac{\varepsilon(t)}{\bar{x}_{r e f}}\right|=\frac{A}{2+2 T^{2} \omega^{2}} \sqrt{\left(\begin{array}{l}
\left(T \omega+T \omega \cos \left(\frac{\pi \omega}{\omega_{1}}\right)+\cos \left(\frac{\pi \omega}{\omega_{1}}\right) \sin \left(\frac{\pi \omega}{\omega_{1}}\right)+\sin \left(\frac{\pi \omega}{\omega_{1}}\right)\right)^{2} \\
+\left(1+\cos \left(\frac{\pi \omega}{\omega_{1}}\right)+T \omega \sin \left(\frac{\pi \omega}{\omega_{1}}\right)+T \omega \cos \left(\frac{\pi \omega}{\omega_{1}}\right) \sin \left(\frac{\pi \omega}{\omega_{1}}\right)\right)^{2}
\end{array}\right)}
$$


and the cycle time is $T_{f}=\frac{1}{2}\left(\sqrt{\frac{16 \bar{x}_{\text {ref }}}{A}}+T_{1}\right)$, which is much longer than precedingly, and comparable to the Bang-bang of Jerk cycle time.

\section{Experimental comparisons of path planning laws}

\subsection{Material and methods}

The experimental validations are carried out on a 3-axes robot (Fig. 8). It has been equipped with a real-time "dSPACE 1103" control card. The available measurements on the motor part come from the actuator axis encoders [10]. When the horizontal axis is only moving, it will be assumed that the axis stiffness remains almost constant (with an actual variation of $30 \%$ ) and the system can be considered as linear. The validation was undertaken for a displacement on the $\mathrm{X}$ axis, with $x_{2}$ varying from $x_{2_{0}}=0$ to $x_{2}=900 \mathrm{~mm}$ and $y_{2}=0 \mathrm{~mm}$ with a height $z=315 \mathrm{~mm}$. According to experimental results, the natural

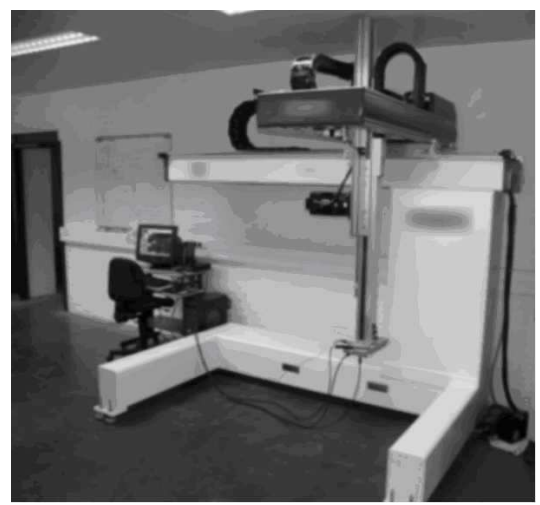

Figure 8: Overview of the first test-setup prototype (stroke [mm]: X-1000 Y-400 Z-800, max. feedrate: $120 \mathrm{~m} \cdot \mathrm{min}^{-1}$, max. acceleration: $4 \mathrm{~m} \cdot \mathrm{s}^{-2}$ ).

frequency was calculated as $F_{n}=9.8 \mathrm{~Hz}$, the damping ratio was taken as $\varsigma=0.0132$. In this case, the optimal value of the jerk given by equation (6), which limits the amplitude of vibrations, is of $30 \mathrm{~m} \cdot \mathrm{s}^{-3}$. Parameters of the industrial control loop were $k_{p}=14 \mathrm{~s}^{-1}, k_{v}=2 \mathrm{~A} . \mathrm{rd}_{\mathrm{s}} \mathrm{s}^{-1}$.

\subsection{Experimental results}

One can see that, from Fig. 9, the curve follows a sinc(.) evolution. Fine jerk tuning is indeed effective. When the jerk parameter is not well-tuned, the vibrational behavior deteriorates slightly. As for the ZVD shaper (Fig. 10), one can find out what was simulated, one obtains zero vibration when $a=\omega_{1} / \omega$ is even, and the curve is flat around points cancelling vibrations. Results for the ZV shaper are alike and not shown here.

\subsection{Comparison and discussion}

One can compare experimentally the maximal error for the three laws: Figure 11 shows that the $\mathrm{ZV}$ shaper is not robust at all. The jerk law is quite robust with respect to input shapers, because the jerk is a continuous derivative of the acceleration during the whole motion, whereas the ZVD cancels vibrations only at the point where zero vibrations are desired. For a value of $a=1$, the three curves have the same percentage of residual vibration (same performances). When $a=0$, one finds again the classical bang-bang in acceleration for which by definition $100 \%$ vibrations occur (Fig. 11). The cycle time is 


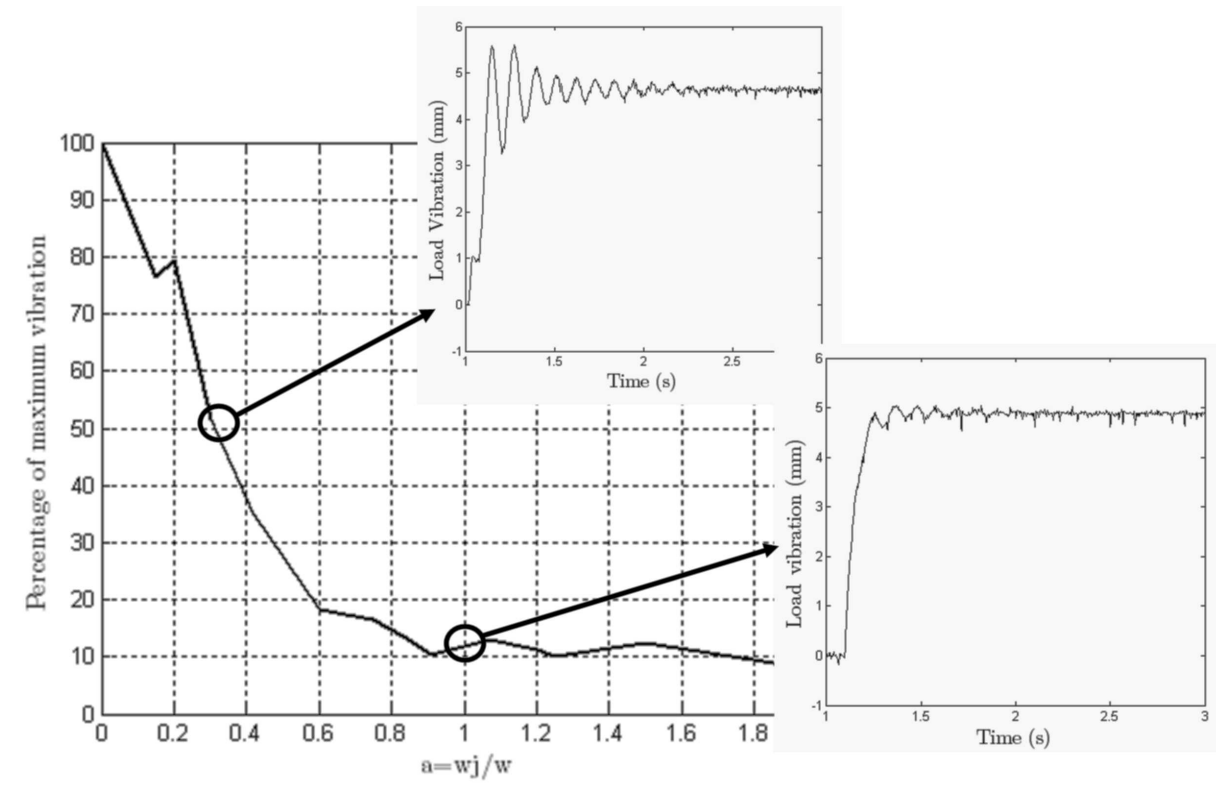

Figure 9: Jerk-limited residual vibrations (\%)-(a) : variation of maximal residual vibrations as a function of $a=\omega_{j} / \omega, \omega_{j}=\frac{2 \pi}{T_{j}}$ (b) : amplitude of vibrations at jerk $\mathrm{J}=100$ (c) : amplitude of vibrations at jerk $\mathrm{J}=30$

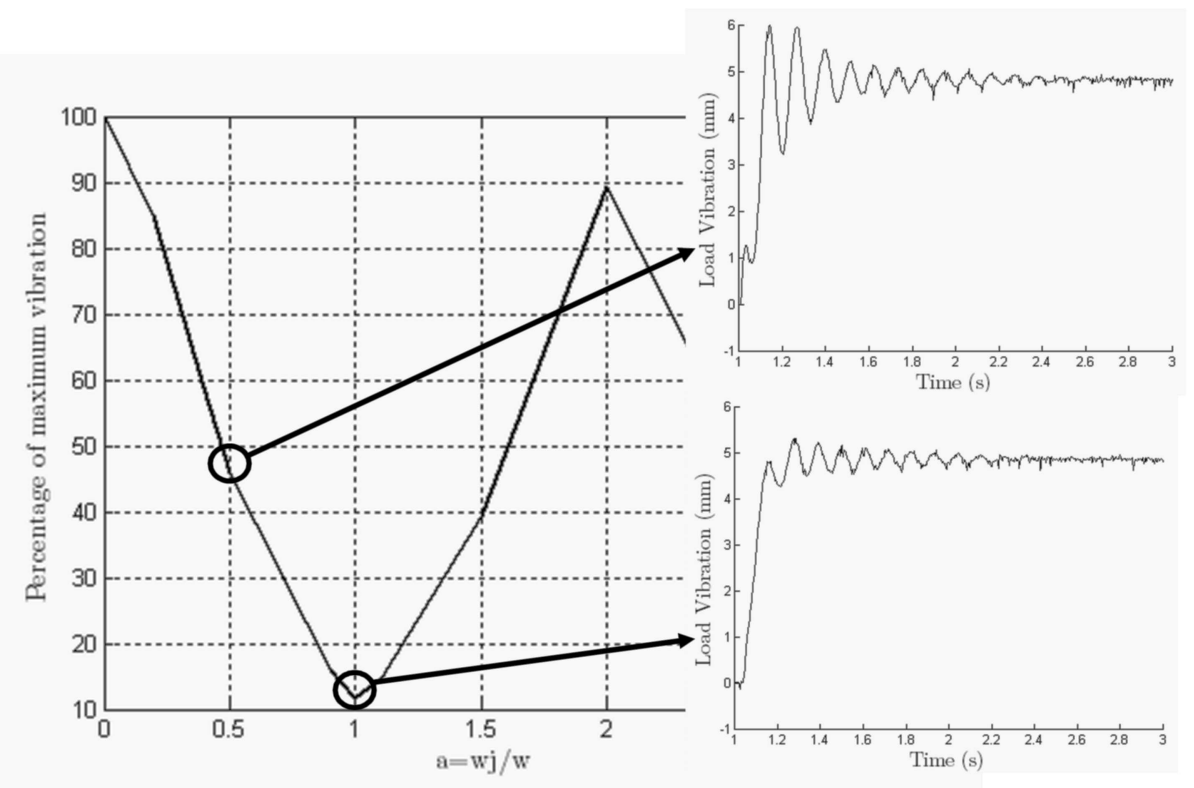

Figure 10: ZVD residual vibrations (\%)-(a) : variation of maximal residual vibrations as a function of $a=\omega_{1} / \omega$, (b) : amplitude of vibrations at $a=0.5$ (c) : amplitude of vibrations at $a=1$ 


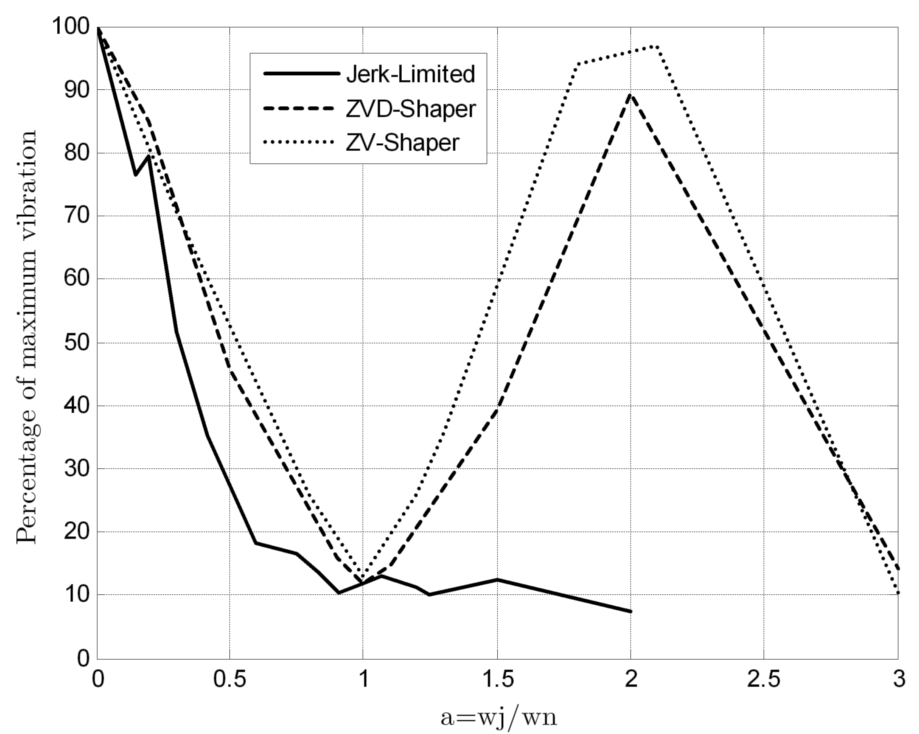

Figure 11: Comparison of planning laws: percentage of maximum vibrations

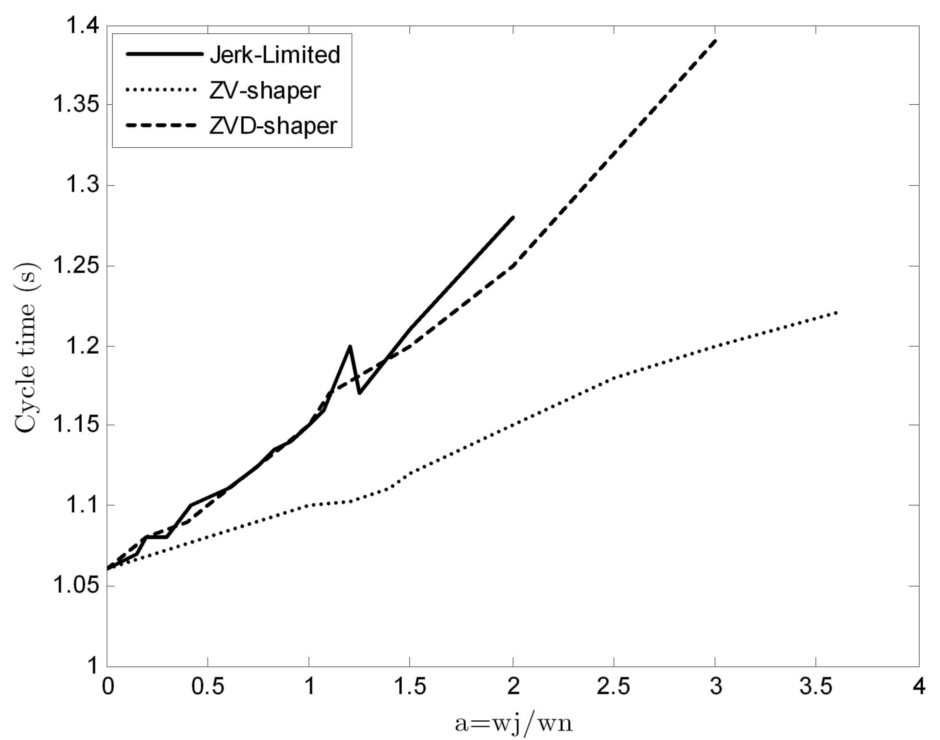

Figure 12: Comparison of planning laws: cycle time 
defined as the instant for which the motor position reaches its reference by $-0.2 \mathrm{~mm}$. Figure 12 shows the evolution of the cycle time of the different laws. One can see that the ZV shaper is quicker by $13 \%$.

As a conclusion, the Jerk bang-bang algorithm is more robust to parameter variations than a $\mathrm{ZV}$ shaper which has approximately the same cycle time. Another advantage is that this algorithm is now implemented in most industrial CNC control devices. When the frequency is always the same, fixed and well-estimated, one can expect to gain some productivity using a ZV shaper instead. A possibility to cope with non-linearities consists either of using "gain scheduling" or adaptive/iterative algorithms e.g. $[11,12]$.

\section{Summary and Conclusions}

The design and computation of near-optimal reference trajectories for CNC devices is a difficult challenge which should examine the trade-off between cancellation of undesirable vibrations and rapidity. This design can be achieved using continuous "jerk" (derivative of acceleration) laws or input shapers, which consist of convolving pulses with the control input. Theoretical and empirical results show the relevance of using jerk laws due to their productivity performances at comparable robustness properties (with respect to natural frequency uncertainties). A good calibration of the model parameters would allow to use a two-pulse shaper, which is faster but less robust. In practice, however, these algorithms have to be adapted to be implemented into CNC units, because of neglected dynamics (such as dry friction) which may cause static errors.

\section{References}

[1] Béarée, R., Barre P.J., Bloch S., Influence of high feed rate machine tool control parameters on the contouring accuracy, J. Intell. Robotic Systems, Vol. 40, 2004, pp 321-342.

[2] Barre P.-J., Bearee R., Borne P., Dumetz, E., Influence of a Jerk Controlled Movement Law on the Vibratory Behaviour of High-Dynamics Systems, J. of Intell. Robot. Systems, Vol. 42, 2005, $275-$ 293.

[3] Fliess M., Lévine J., Martin P., Rouchon, P., Flatness and defect of nonlinear systems: Introductory theory and applications, Int. J. Control, Vol. 61, 1995, pp 1327-1361.

[4] Singer N., Seering W., Preshaping command inputs to reduce system vibration, J. Dynam. Syst., Meas. Contr., Vol. 112, 1990, pp 76-82.

[5] Meckl P. H., Seering W. P., Experimental evaluation of shaped inputs to reduce vibration of a cartesian robot, J. of Dyn. Systems, Meas. Control, Vol. 112, 1990, pp 159-165.

[6] Peláez G., Gu. Pelaez, J.M. Perez, A. Vizán, E. Bautista, Input shaping reference commands for trajectory following Cartesian machines, Control Eng. Practice, Vol. 13, 2005, pp 941-958.

[7] Singhose W., Singer N., Seering W., Time-optimal negative input shapers, J. Dynam. Syst., Meas., Contr., Vol. 119, 1997, pp. 198-205.

[8] Lau M. A., Pao, L. Y. , Input shaping and time-optimal control of flexible structures, Automatica, Vol. 39, 2003, pp 893-900.

[9] Singhose W. E., Derezinski S. J., Singer N. C., Extra insensitive input shaper for controlling flexible spacecraft, J. Guidance Control Dynamics, Vol. 19, 1996, pp 385-391. 
[10] Dumetz E., Dieulot J.Y., Barre P.J., Colas F., Control of an Industrial Robot using Acceleration Feedback, J. Intell Robotic Systems, Vol. 46, 2006, pp 111-128.

[11] Md Zain M.Z., Tokhi M.O., Mohamed Z., Hybrid learning control schemes with input shaping of a flexible manipulator system, Mechatronics, Vol. 16, 2006, 209-219.

[12] Cutforth, C. F., Pao L. Y., Adaptive input shaping for maneuvering flexible structures, Automatica, Vol. 40, 2004, pp 685-693.

Jean-Yves Dieulot

ENSAM de Lille

Research Technological Team CEMODYNE

8 bd Louis XIV, 59000 LILLE Cedex

E-mail: jean-yves.dieulot@ polytech-lille.fr

Received: November 6, 2006 